\title{
Study design VII. Randomised controlled trials
}

\author{
Kate Ann Levin \\ Child and Adolescent Health Research Unit, University of Edinburgh , Edinburgh, Scotland, UK
}

\begin{abstract}
Previously in this series, I have given an overview of the main types of study design and the techniques used to minimise the likelihood of obtaining biased results. In this article I describe more fully randomised controlled trials, their uses, advantages and limitations.

Evidence-Based Dentistry (2007) 8, 22-23. doi:10.1038/sj.ebd.6400473
\end{abstract}

The study designs described previously in this series have focused on observational studies, where outcome measures have been recorded either at one specific timepoint (cross-sectional) or repeatedly over time (longitudinal). Information may be collected at an aggregate level (ecological) and may be gathered prospectively (cohort study) or retrospectively (case-control study).

Randomised controlled trials (RCT), or randomised clinical trials, are experimental studies where the effect of an intervention is assessed by collecting data before and after an intervention has taken place. RCT are used to compare an intervention with one or more other interventions or with no intervention. Interventions are often clinical treatments but may also be educational interventions such as health promotion leaflets.

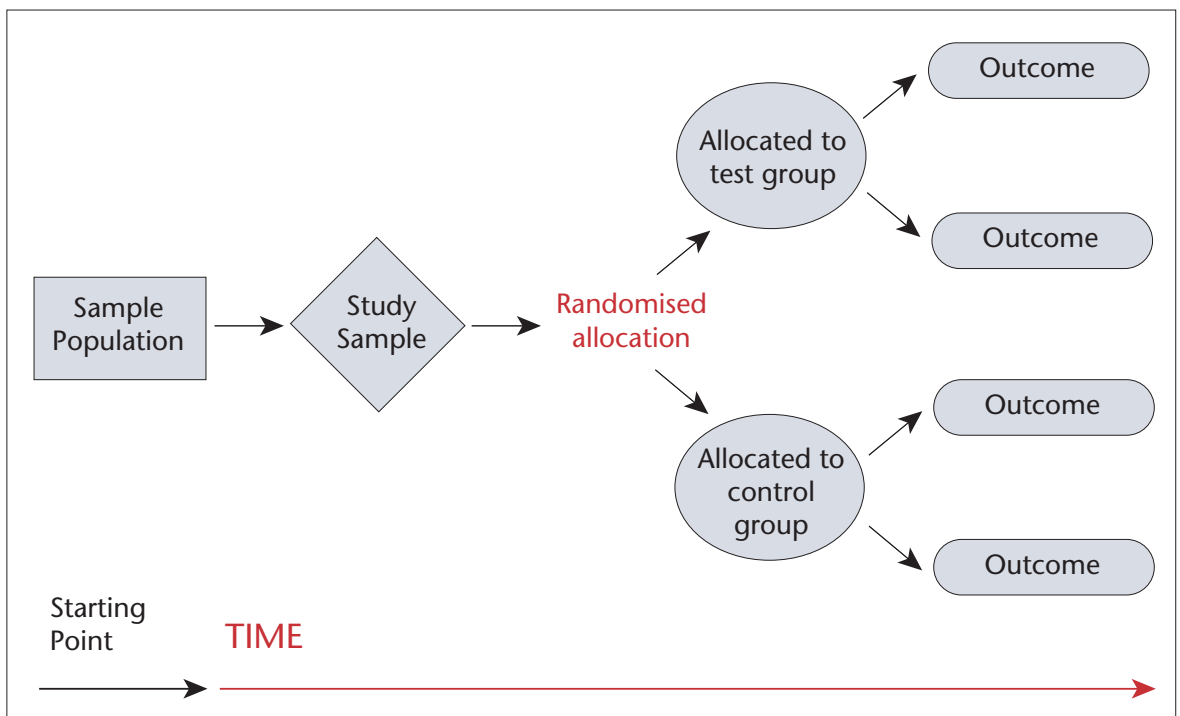

Figure 1. Diagrammatic representation of a randomised controlled trial
What are the two main features of the RCT?

\section{They are comparative}

In RCT, an intervention is investigated by comparing one group of people who receive the intervention with a control group or control arm who do not (Figure 1, below). The control group receives the usual or no treatment and their outcome measure, or the change in measure from the starting point or baseline, is compared with that of the intervention group.

\section{They are designed to minimise bias Allocation bias}

Allocation bias occurs when the measured treatment effect differs from the true treatment effect because of how participants were selected into the intervention or control groups. In RCT, once the participants are entered into the study, they are randomised to either an intervention group or the control group. Randomisation ensures that characteristics that might affect the relationship between intervention and outcome measures will be roughly equal across all arms of the study, minimising potential bias.

\section{Performance bias}

Even after randomised allocation, bias can occur. Performance bias occurs when participants' response to the treatment is affected by knowledge of the group to which they are assigned, or when health professionals administer treatments differently between treatment arms.

\section{Assessment bias}

Health professionals assessing the outcome of treatment relative to alternative or placebo interventions may record outcome measures biased by the knowledge of the group to which the participant has been assigned. Over- (or under-) estimation of the effects of an intervention, even if subconscious, is known as assessment bias. Alternatively there might be a systematic difference in measuring the outcomes between the two groups because of the method of recording used. For example, where the control group is assigned to one practitioner and the intervention group to another, or where groups are assessed at different times of the day, there may be a systematic difference between groups in the mean outcome measure recorded. Bias will be minimised where a standardised method of evaluation is used across both groups. Subjective 
measures to assess the effectiveness of a treatment will be more prone to bias.

\section{Attrition bias}

Attrition bias (also call loss-to-follow-up bias) occurs when patients drop out of the study from one or other of the study groups preferentially. For example, if halfway through a study the treatment has been successful participants may drop out, and information about the success of the treatment is then lost. Conversely, participants in the control group may be unhappy with their lack of progress and may drop out of the study in order to seek alternative help.

\section{Allocation concealment}

Bias will be minimised where the allocation schedule is concealed of whom is assigned to which group. Blinding (also known as masking) helps to prevent systematic differences between comparison groups in prognosis or responsiveness to treatments (allocation bias). Blinding of both participants and practitioners prevents performance and assessment bias by ensuring that participants, treatment administrators and those measuring outcomes do not know which treatment was given. Where possible, it is recommended that RCT participants are blind to the treatment they receive. In order to do this it is sometimes appropriate for the control group to receive a placebo. This enables the RCT to be blind and overcomes the placebo effect, where it is the action of taking medication and not the medication itself that results in a positive outcome.

\section{Why carry out RCT?}

RCT are prospective longitudinal studies, allowing the investigation of causal associations between interventions and outcomes. The random selection of participants into each arm and the controlled way in which the trial is carried out mean that all factors other than the intervention are considered equal. Although associations may be investigated under observational studies, causality cannot be inferred. If possible, it is always preferable to choose RCT over other study designs when assessing the impact of one or more interventions.

\section{Factors to be considered when carrying out RCT Sample size}

The size of the sample required when carrying out RCT is dependent upon the power of the test and what size of intervention impact is considered meaningful. It also depends on the type of hypothesis the RCT is testing. The smaller the magnitude of difference between groups that is to be detected and the greater the variability in outcomes, the larger the sample size that will be required.

\section{Stratification}

Very large trials are likely to have a good balance of patients within each arm. When the samples are small, however, treatment groups may by chance end up with different characteristics, which may affect the outcome of the trial. Stratification is a way of ensuring the treatment groups are balanced on characteristics that are likely to alter the relationship between treatment and outcome.

\section{Crossover design}

Crossover trials are another way of overcoming differences in groups by keeping the patients as matched as possible. Instead of having different patients in each treatment group, patients receive first one treatment and then the other, in a random order, with a washout period in between. Withinpatient differences are then compared. Thus each patient effectively becomes their own 'test' and 'control'.

\section{Between group contamination}

Educational interventions, in particular, are prone to contamination where, for example, a member of the control arm is a friend of a patient receiving the oral health advicesheet intervention. Information may then pass between the two arms of the trial, and thus alter the results.

One way to overcome this is to use cluster sampling so that natural clusters such as geographic areas or dental practices are randomised rather than individuals.

\section{Ethical issues}

RCT are not always possible because of ethical issues when assigning patients to study arms.

If one group of patients receives treatment thought to be effective, while another group does not, the ethics of a trial may be brought into question. Similarly, there are some trials that cannot be carried out because they may actively encourage unhealthy practices such as smoking; people cannot be randomised into smoking and nonsmoking groups.

\section{Analysis of data}

RCT are experiments set up to test hypotheses. The null hypothesis (H0) is that the intervention will have no impact on the outcome measure (ie, that the outcome of interest will be similar in both the test and control groups). The alternative to the null hypothesis (H1) is that the intervention will have a meaningful effect and that this effect will be statistically significant. The statistical method required to test this hypothesis will depend on the nature of the outcome of interest. Comparing the proportions of patients achieving a 'successful outcome' between treatment groups (eg, those satisfied with their treatment) will require a different approach from investigating average differences between groups (eg, average probing pocket depths).

\section{Advantages of RCT}

- Ability to make causal inferences mean that RCT provide the strongest empirical evidence of a treatment's efficacy.

- Randomisation of participants to the test and control arms and concealment of their allocation ensures that allocation bias and confounding of unknown variables are minimised.

- The study can be tailored to answer a specific research question.

\section{Disadvantages of RCT}

- High dropout when the intervention has undesirable side-effects or there is little incentive to stay in the control arm.

- Ethical considerations may mean that a research question cannot be investigated using the RCT design.

- For a descriptive overview it may be cheaper and easier to use an observational design.

- Prior knowledge is required about the level of improvement that is clinically meaningful and the expected variation of improvement in the sample in order to calculate the RCT sample size. These facts are often not known.

\section{Recommended reading}

1. Pocock SJ. Clinical Trials: a Practical Approach. Chichester: Wiley; 1983.

2. Altman DG, Bland JM. Treatment allocation in controlled trials: why randomise? Br Med J 1999; 318:1209.

3. Begg C, Cho M, Eastwood S, et al. Improving the quality of reporting randomized controlled trials: the CONSORT statement. J Am Med Assoc 1996; 276:637-639. 